\title{
Cinco décadas de linguística feminista: índices de consolidação do campo
}

\author{
Amanda Diniz Vallada' (iD 0000-0002-5512-6087 \\ Joana Plaza Pinto' [i] 0000-0001-8052-9390 \\ 'Universidade Federal de Goiás, Goiânia, GO, Brasil. \\ 74690-900 - ppgllufg@gmail.com
}

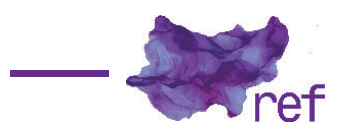

\begin{abstract}
Resumo: Neste artigo, buscamos descrever as características da literatura sobre linguagem e gênero que influencia as pesquisas da linguística feminista publicadas em artigos pós-anos 2000, cinco décadas depois do estabelecimento do campo, em 1975. Estudamos esses mesmos artigos quanto ao seu posicionamento explícito acerca da relevância do gênero para a pesquisa em linguística feminista. Para tanto, selecionamos artigos anglófonos e brasileiros, publicados de 2000 a 2017. Os resultados indicam que o conjunto de publicações mais citadas como apoio teórico dos artigos analisados é o de autoria da linguista britânica Deborah Cameron. Já a obra individual mais consultada trata-se de Gender of Trouble, de Judith Butler (1990). Sobre a relevância de questões de gênero para a pesquisa, percebemos que os artigos assumem como certa sua importância, o que pode ser tomado como um índice da consolidação do campo.
\end{abstract}

Palavras-chave: linguística feminista; pesquisa feminista; gênero; linguagem; discurso.

Five Decades of Feminist Linguistics: Field Consolidation Indexes

Abstract: In this article, we search to find out, five decades after the field's foundation in 1975, the main language and gender literature, which has been influencing post 2000 articles of Feminist linguistic researches. We studied these same articles regarding their explicit positioning about the relevance of the gender for feminist linguistics research. For that, we selected articles produced in Anglophone and Brazilian contexts, published from 2000 to 2017. The results suggest that the set of publications most cited as theoretical support by the analysed articles is those of the British linguist Deborah Cameron. The most individually consulted work is Judith Butler's Gender Trouble (1990). Regarding the relevance of gender issues for research, we notice that most of the articles take its importance for granted, which can be taken as an index of the consolidation of the field. Keywords: Feminist linguistics; Feminism; Gender; Language; Discourse.

\section{Introdução}

A pesquisa registrada neste artigo tem como proposta atentar para os estudos de linguística feminista, cujo marco inicial eleito aqui é a publicação da obra Language and Women's Place, de Robin Lakoff (1975). O mérito de Lakoff é inegável, pois, tendo sido lançada no ano em que a Assembleia Geral da ONU declarou como Ano Internacional das Mulheres e organizou a primeira Conferência Mundial sobre as Mulheres, sua obra inaugurou a possibilidade de se estudar as práticas linguísticas das mulheres com a mesma importância com que se estudavam as práticas linguísticas de outros grupos sociais. Mas também foi um pouco além, ao politizar seus estudos e legitimar os incômodos da linguagem como pauta feminista. Ainda que sua filiação inicial fosse com a sociolinguística, desde então vêm-se desenvolvendo, em vários campos da linguística, pesquisas que entendem questões de gênero como centrais para lidar com questões de linguagem, e vice-versa. Mesmo que nem todas as pesquisas sobre gênero e linguagem se filiem a epistemologias feministas, certamente seria possível chamar esse campo de linguística feminista. 
Considerando esse marco, a linguística feminista está em sua quinta década de atividade e os trabalhos que alinham gênero e linguagem já tiveram oportunidade para se configurar relativamente estabelecidos como campo de produção de conhecimento científico. Diante disso, imaginamos que houvesse uma consolidação do campo e propusemos este estudo para identificar algumas características atuais da linguística feminista em publicações anglófonas e brasileiras.

Desenhamos, assim, uma pesquisa bibliográfica com as seguintes perguntas: 1) Quais são as obras clássicas ${ }^{1}$ da linguística feminista, produzidas a partir do marco de 1975, que influenciam os artigos do campo a partir dos anos 2000? 2) Há nos artigos pós-anos 2000 uma preocupação em estipular qual é a relevância do gênero para estudar aspectos da linguagem?

A primeira pergunta objetiva identificar algumas características atuais do campo por meio de suas referências clássicas, que vêm influenciando os artigos da linguística feminista a partir dos anos 2000. A segunda pergunta parte da hipótese de que, depois de cinco décadas, o campo já estaria suficientemente consolidado e, para checar esta hipótese, examinamos os artigos publicados a partir dos anos 2000 quanto ao seu posicionamento sobre o papel do gênero para a pesquisa em linguística.

Para apresentar o detalhamento e resultados da pesquisa, organizamos este artigo em quatro seções. Na primeira seção, apresentamos o pano de fundo do campo de interesse desta pesquisa, seus precedentes teóricos em convergência com as posições teóricas mais recentes, incluindo uma descrição da construção da linguística feminista, as divisões mais conhecidas dos estudos de linguagem e gênero e a perspectiva de que gênero é uma construção social que se dá pela linguagem. Na segunda seção, descrevemos os procedimentos adotados para a seleção dos artigos de linguística feminista publicados após os anos 2000 que são contemplados pela análise. Na terceira seção, buscamos, nas referências dos artigos selecionados, as obras citadas que tratam sobre linguagem e gênero e influenciam as produções atuais, e os temas que estão articulados a essa produção. A parte final do artigo consiste na análise sobre como esses estudos se posicionam acerca da relevância das questões de gênero para o campo da linguagem. Nossas conclusões retomam as análises para avaliar como os artigos analisados impactam o campo da linguística feminista.

\section{A constituiçāo de um campo de estudos de linguagem e gênero}

Desde sua publicação, em 1975, Language and Women's Place, de Lakoff, tem sido visto, conforme Mary Bucholtz (2004, p. 3), "como o começo dos estudos de linguagem e gênero como um subcampo da linguística, e como a inauguração dos estudos de linguagem e gênero em disciplinas relacionadas [...]". ${ }^{2}$ Além de examinar "a linguagem usada por e sobre as muIheres", Lakoff pretendia prover evidências linguísticas sobre a desigualdade entre os papéis de mulheres e homens (LAKOFF, 1975, p. 1).

Na edição de 2004 da mesma obra, Lakoff lista mais três de seus objetivos, e separa seus objetivos como linguista de seu objetivo como feminista. A Lakoff linguista (2004, p. 19, grifo da autora) se propôs a demonstrar "que ao menos um artefato extralinguístico (no caso, gênero) exigia representação linguística" e "que gênero exigia representação linguística até mesmo em línguas como o inglês, em que sua presença era (talvez) menos percebida do que em línguas "exóticas'"'3. A Lakoff feminista, por sua vez, queria "usar as discrepâncias linguísticas entre muIheres e homens como um diagnóstico das desigualdades sociais e psicológicas entre os sexos".

Além da obra de Lakoff, outros trabalhos iniciais da sociolinguística que focalizavam a questão de gênero, como os de Don Zimmerman e Cadence West (1975), Pamela Fishman (1983) e West e Zimmerman (1983), presumiam que o gênero deveria ser estudado nas situações em que fosse mais evidente, isto é, nas interações estabelecidas entre falantes de diferentes sexos com possibilidade de se envolverem em uma relação sexual (Bonnie McELHINNY, 2003).

Seguindo a mesma tendência de muitos outros campos de estudos feministas das décadas de 1970 e 1980 (Adriana PISCITELLI, 2002), o dualismo sexo/gênero presente nos primeiros estudos sociolinguísticos, como entende McElhinny (2003), motivou essa recomendação metodológica. Nessa noção dualista, conforme Nicole-Claude Mathieu (2005, p. 44), "frequentemente, pensa-se que sexo emerge da 'biologia', ao contrário de gênero, que é visto como 'social'", uma dicotomia debitaria do importante texto de Gayle Rubin (1975) sobre o sistema sexo/gênero. O que podemos notar, neste caso, não chega a ser uma surpresa: a linguística feminista dos anos 1970 se constituiu com as mesmas tendências de outros campos próximos, como é o caso de Rubin (1975) na antropologia, e se fortalece nos anos 1980, pressupondo essa dicotomia sexo/gênero nas análises.

'Definiremos esta noção de "clássica" mais adiante.

2 Todos os trechos citados em inglês foram traduzidos pelas autoras para fins exclusivos desta publicação.

${ }^{3}$ As línguas exóticas a que Lakoff se refere seriam o japonês, o arawak, o dyirbal e o koasati, línguas que exigiam que o gênero dos/das participantes e sujeitos da conversação fosse linguisticamente codificado. 
Esse dualismo foi bastante criticado pelas feministas antiessencialistas nas décadas seguintes (PISCITELLI, 2002), especialmente a partir do livro de Judith Butler (1990) e sua crítica produtiva ao sistema sexo/gênero. Aplicar a metodologia articulada gerada nesse dualismo implica estudar gênero somente nas interações heterossexuais, o que pode ser falacioso, já que diferenças de gênero podem apenas ser exageradas nesses contextos (McELHINNY, 2003).

Para superar as implicações dessa metodologia dualista, desde Butler (1990), pesquisadoras/es feministas vêm adotando uma abordagem que coloca sobre o sexo o olhar que estava sobre o gênero. Três modos de entender a relação entre sexo e gênero foram discriminados por Mathieu (2005): Modo I - sexo e gênero são homólogos; gênero é a expressão social da biologia individual determinada pelo sexo; Modo II - sexo e gênero são análogos; gênero simboliza sexo em uma experiência coletiva (padrões sociais são impostos em razão da biologia); Modo III - sexo e gênero são heterogêneos; gênero não é a expressão nem o símbolo social de sexo.

Assim, estudiosas/os fora da perspectiva dualista atentam-se à construção social de sexo e gênero; às diferenças culturais e sociais acerca do corpo; às hegemonias, contestações e implicações das definições de gênero e sexo nas/pelas sociedades; e às motivações políticas para a normativização de certas definições. Essa abordagem feminista antiessencialista chamada pós-estruturalista (ou "pós-moderna", em algumas discussões; cf. BUTLER, 1998) refletiu, na linguística, em estudos que focavam nas transgressões às normas de sexo, gênero e identidade sexual, como os estudos de Kira Hall (1997) sobre as hijras indianas, e de Deborah Cameron (1997a) sobre as fofocas em que estudantes universitários se engajam.

Como já é bastante divulgado, os estudos de linguagem e gênero podem ser organizados de acordo com seu tipo de abordagem (CAMERON, 2005b, Ana Cristina OSTERMANN, Beatriz FONTANA, 2010). ${ }^{4}$ Os estudos que se enquadram na abordagem do déficit sugerem que a maneira como as mulheres falam é deficiente se comparada à maneira como os homens falam. Nessa perspectiva estão as concepções de Otto Jespersen (2005) e Robin Lakoff (1975) sobre as práticas linguísticas das mulheres. Para o autor, às mulheres cabem os eufemismos e "refinamentos". Aos homens, os "chefes renovadores da linguagem", cabe uma linguagem com vigor e franqueza. Contudo, ao passo que Jespersen (2005) considera que essa deficiência seria predominantemente natural, isto é, resultado de uma incompetência da natureza das mulheres, Lakoff (1975) sustenta apenas que os recursos linguísticos das mulheres têm status inferior, sem considerar uma suposta incapacidade natural.

Na abordagem da dominância, as pesquisas representadas em West e Zimmerman (1983) apontam que essas diferenças na linguagem seriam causadas pela posição subordinada das mulheres em relação aos homens. Já a abordagem da diferença sugere que as diferenças linguísticas entre homens e mulheres refletiriam as normas sociais e linguísticas de suas diferentes culturas (CAMERON, 2005b). Na perspectiva da diferença estão estudos da sociolinguística interacional (Deborah TANNEN, 1990; 1999), a qual entende que as regras de interação são próprias de cada grupo cultural, e podem causar confusões quando pessoas de diferentes grupos interagem.

Enquanto os estudos de déficit não têm mais espaço na pesquisa feminista em linguagem e gênero, a dominância e a diferença ainda geram debate dentro do campo. De um lado, pesquisadores/as da abordagem da dominância são criticados/as, "por fazerem suposições simplistas a respeito da intenção dos homens de dominar as mulheres na conversação e a respeito da relação entre estratégias linguísticas e (falta de) poder" (CAMERON, 2005b, p. 15). Do outro, pesquisadoras/es da diferença são crificadas/os pela ênfase que dão à diferença nas interações entre mulheres e homens de forma generalista, sendo incapazes de distinguir situações em que um homem, como indivíduo, quer dominar uma mulher, e chamando conflitos entre pessoas em desigualdade de "mal-entendidos" (BUCHOLTZ, 2003; CAMERON, 1998a; 2005b). Além disso, é um erro que os/as proponentes da diferença presumam que poder e cultura sejam construções independentes (Aki UCHIDA, 2005).

Esses tipos de abordagens se apresentam em versões essencialistas e antiessencialistas, ainda que estas últimas tenham se fortalecido nos estudos feministas em geral na década de 1990 (cf. Jane GALLOP, 1997), ${ }^{5}$ assim como nos estudos de linguagem. Mas esse não foi o único aspecto sob escrutínio nessa década da linguística feminista. Na sociolinguística e na linguística antropológica, linguistas seguiram pensando "gênero como um atributo", uma qualidade individual:

\footnotetext{
${ }^{4}$ Apesar de reconhecer essas abordagens, Cameron (2005b) prioriza a organização dos estudos feministas de linguagem por temas principais e defende fortemente a sobreposição entre eles, argumentando que, apesar de discordâncias significativas, há um diálogo produtivo, com preocupações compartilhadas, mas diferindo nas posições feministas.

5 Jane Gallop (1997), ao observar esta tendência antiessencialista, confronta uma separação estanque entre feministas antes e depois de 1990 e problematiza as críticas reducionistas das complexidades dos estudos feministas anteriores aos anos 1990.
} 
Frequentemente, antropólogas/os trabalhando dentro de uma tradição ocidental continuam a usar a lógica de commodity para compreender gênero. Continuam a se fascinar pelos atributos das coisas, e a identificar posse, propriedade, controle, em uma relação íntima entre os atributos discretos e o indivíduo uniforme (McELHINNY, 2003, p. 28).

Porém, como McElhinny (2003) aponta, superar a ideia de gênero como posse tem sido um desafio aceito por pesquisadoras/es do ocidente, em especial dos Estados Unidos e da Europa Ocidental. Nessa perspectiva, o livro de Marjorie Goodwin (1990) sobre meninas e meninos afro-americanas/os em várias brincadeiras e atividades de fala traz a noção de "gênero como atividade". Conforme o contexto, uma mesma criança constrói gênero de modos diferentes, ou seja, a identidade de gênero varia quando a criança muda de uma atividade para a outra.

Além do conceito de atividade de Goodwin (1990), estudos de sociolinguística também são norteados a partir da ideia de "gênero como prática". O conceito de "comunidade de prática", introduzido nos estudos de linguagem e gênero por Penelope Eckert e Sally McConnell-Ginet (1992), refere-se a uma construção social em que pessoas se engajam em torno de um empreendimento comum, do qual emergem práticas geradas pelas atividades conjuntas dessas pessoas.

Embora tanto Goodwin (1990) quanto Eckert e McConnell-Ginet (1992) tentem superar e criticar o essencialismo de gênero como categoria de análise, suas ideias de gênero como prática e de gênero como atividade têm diferentes implicações, uma vez que a ideia de gênero como prática nos permite enxergar que essas práticas ocorrem dentro do contexto de estruturas culturais ou institucionais, enquanto gênero como atividade pode dirigir o foco da pesquisa somente ao gênero individual, o que desconsidera o papel do gênero nas instituições, bem como atribui a falantes uma grande agentividade nas suas escolhas de linguagem e na construção de suas identidades sociais (McELHINNY, 2003).

Quando pesquisadoras/es em linguagem e gênero não querem estudar (somente) o papel do gênero nas interações informais entre homens e mulheres, a atenção da pesquisa se volta para como "as diferenças de gênero são criadas, por exemplo, na separação entre trabalho remunerado e não-remunerado, na segregação de locais de trabalho, na criação de 'trabalho de homem' e 'trabalho de mulher'” [...] (McELHINNY, 2003, p. 32), pois

como certas estratégias linguísticas estão indiretamente e indexicalmente ligadas a certos grupos, instituições precisam apenas ser organizadas para definir, demonstrar e reforçar a legitimidade e a autoridade das estratégias linguísticas associadas a um gênero, enquanto negam o poder de outras estratégias de excluir um grupo sem a necessidade de tornar a exclusão explícita.

Além da relevância do gênero na organização de instituições, o papel do conceito de gênero na pesquisa foi especialmente abordado por analistas da conversa como Zimmerman e West (1975) e Fishman (1983) que, após estudos declaradamente feministas, passaram a se preocupar em rejeitar motivações políticas no campo da análise da conversação, sobretudo após as críticas de Emmanuel Schegloff (1997; 1999) (BUCHOLTZ, 2003).

No seu artigo de 1999, Schegloff afirma que a relevância de gênero e outras identidades sociais não pode ser presumida por analistas a partir de suas posições políticas, mas devem estar explicitamente presentes na interação a ser analisada. Ou seja, não devem ser relevantes para quem pesquisa, mas para as/os participantes da fala-em-interação. Como interpreta Bucholtz (2005), embora Schegloff não exclua completamente a possibilidade de uma análise da conversa baseada em gênero, suas restrições são rigorosas a ponto de limitar esse tipo de análise a interações em que o gênero seja excessivamente topicalizado.

Várias analistas da conversa, como Ann Weatherall (2000; 2002) e Margaret Wetherell (1998), têm uma abordagem contrária à de Schegloff, rejeitando suas premissas e/ou articulando-as com uma postura mais crítica. Para Weatherall (2000, p. 287), "índices linguísticos de gênero podem acontecer em todo nível de linguagem. Assim, mesmo que o gênero não seja explicitamente considerado pelas/pelos participantes como relevante na conversa, é um traço onipresente nas interações".

Sobre as possíveis abordagens da análise da conversa, Bucholtz (2003, p. 53) percebe que:

A diversidade de usos e críticas feministas sobre a análise da conversação deixa evidente que a questão acerca dos limites de uma abordagem desse campo sobre gênero está longe de ser resolvida. Ainda assim, toda a gama de analistas da conversação compartiIha de uma mesma perspectiva de gênero como um fenômeno cujo sentido e relevância devem ser analiticamente fundamentados nas próprias concepções das/dos participantes sobre a interação e não contrabandeados na análise por meio dos compromissos e pressuposições da pesquisadora ou do pesquisador.

Se, na análise da conversa, estudiosas/os feministas se viram em dificuldades para se estabelecer, na análise crítica do discurso encontraram tudo de que precisavam para prosperar 
como um campo da linguística. A própria noção de "crítica", um posicionamento progressista por parte do/a pesquisador/a que se propõe a intervir na sociedade, baseou pesquisas sobre linguagem e gênero. "Enquanto a análise da conversa insiste que o poder deve ser descoberto na interação e não pode ser o ponto de partida da análise", Bucholtz escreve, "a análise crítica do texto sustenta que o poder permeia todos os aspectos da sociedade e, portanto, é operativo em todo discurso" (BUCHOLTZ, 2003, p. 58).

Com o objetivo de demonstrar esse aspecto do poder nas relações e interações entre os gêneros, estão os estudos de Mary Talbot (1995; 1997; 2000). Em seu texto de 1997, Talbot analisa a descrição de um caso de assédio sexual no trabalho feita por um tabloide britânico que reforça pressuposições tradicionais sobre a masculinidade e as relações entre mulheres e homens. Além desse texto, Talbot $(1995 ; 2000)$ e Val Gough e Talbot (1996) também se voltam àquilo que é central para a análise crítica do discurso: identificar as reversões entre o que um texto de fato faz e o que ele simula fazer (BUCHOLTZ, 2003).

Abordamos nesta seção alguns problemas da construção do campo de estudos feministas de linguagem e gênero. A seguir, tratamos das decisões metodológicas da pesquisa. São apresentados os passos tomados para a seleção dos artigos brasileiros e anglófonos que analisamos a fim de responder às perguntas de pesquisa estabelecidas.

\section{Metodologia}

O portal de periódicos da CAPES (http://www.periodicos.capes.gov.br) foi a principal base para as buscas e seleções dos artigos anglófonos e brasileiros aqui analisados. Para todas as buscas, definimos que os artigos encontrados deveriam ser somente os de periódicos revisados por pares e publicados de 2000 a 2017.

Além deste intervalo de tempo, os artigos foram buscados por mais dois critérios: geo-institucional e linguístico. Aqueles artigos publicados em português cujas/os autoras/es estão vinculadas/os a alguma instituição brasileira foram considerados produção brasileira, assim como foram considerados artigos anglófonos aqueles publicados em inglês cujas/es autoras/es estão vinculadas/os a alguma instituição de um país onde o inglês é língua oficial. Esses critérios foram definidos para encontrar artigos com potencial de circulação no Brasil ou em países de língua inglesa. É evidente que, no contexto atual de políticas (linguísticas e ideológicas) de circulação transnacional do conhecimento, o impacto desses artigos não pode ser medido por tais critérios de busca, portanto, nossa análise não inclui fator de impacto na bibliometria.

É importante destacar que os resultados encontrados pelo portal da CAPES apresentaram alguns problemas. Várias resenhas, entrevistas, editoriais e outros tipos de documentos em outras línguas surgiram das buscas, ainda que tenhamos aplicado devidamente os critérios descritos. Tais documentos foram descartados.

Em todas as buscas, selecionamos os artigos que tivessem em suas palavras-chave ou em seu assunto (categoria indiciada pelo próprio portal) quaisquer um ou mais dos termos "feminismo", "teoria feminista", "gênero" e "estudos de gênero", combinados com qualquer um dos termos "discurso", "análise do discurso", "análise da conversação" e "linguagem"; o mesmo procedimento foi aplicado aos termos em inglês feminism, feminist theory, gender e gender studies, combinados com discourse, discourse analysis, conversation alanalysis e language.

A primeira busca foi feita com os termos "feminismo" e "linguística" e feminism e linguistics, e resultou em 95 documentos encontrados em português e 82 em inglês. Destes montantes, uma parte significativa não continha os dois termos nas palavras-chave, mas sim em outros locais do artigo, incluindo títulos nas referências bibliográficas ou em uma nota etc., não preenchendo os critérios que estabelecemos. Selecionamos então os que continham os dois termos nas palavras-chave. Assim, dentre os artigos em português, apenas o de autoria de Rodrigo Borba e Ostermann (2008) faz parte desta análise. Karyn Stapleton (2001), Nina Philadelphoff-Puren (2004), Bucholtz e Hall (2005) e Frederick Attenborough (2013) foram os artigos anglófonos selecionados.

Foi curioso, neste levantamento inicial, que alguns artigos em português que já conhecíamos não apareceram. Fizemos, então, um teste de busca com os termos em inglês gender, feminism e language para artigos escritos em português, e fomos surpreendidas com artigos que não apareciam na busca com termos em português, mesmo que estes estivessem presentes nas palavras-chave. Essa ocorrência deixa entrever os limites do sistema de busca do Portal de Periódicos, e nos obrigou a testar todos os termos em inglês para artigos em português.

Nos resultados desta nova busca, encontramos 22 artigos. A maioria era resumo de artigos em português ou em outras línguas, alguns repetidos em relação às buscas anteriores. Assim, os artigos de Joana Plaza Pinto e Suzana Badan (2012), Mariléia Sell (2012) e Sell e Ostermann (2009) atendiam aos critérios. O mesmo ocorreu para os termos gênero e linguagem, resultando em treze artigos, dos quais apenas Ostermann (2008) não aparecia em buscas anteriores e continha as duas palavras-chave. 
Em inglês, a combinação dos três termos (feminism, gender, language) gerou 55 resultados. Destes, Sarah Murnen (2000), Talbot (2000), Aneta Pavlenko (2001), Elizabeth Peel (2001), Weatherall (2002), Sara Mills (2003), Mary Crawford e Annie Fox (2007), Clare Stockill e Celia Kitzinger (2007), Kitzinger e Rickford (2007), Cameron (2015) e Weatherall (2015) atendiam aos critérios.

Além do portal da CAPES, fizemos uma breve pesquisa no Google Scholar por "linguística feminista", entre aspas, nas publicações em português entre 2000 e 2017, excluindo as citações. Encontramos 20 resultados, dos quais 17 foram excluídos ao aplicarmos os critérios (não eram artigos, ou eram repetições de artigos já encontrados no portal da Capes). Os três artigos que atendiam a todos os critérios foram: Débora Figueiredo (2009), Izabel Magalhães (2009) e Giselly Andrade, Gislene Carvalho e Romana Zambrano (2017). Vale observar que, apesar da vantagem de terem sido encontrados esses três artigos, o Google Scholar apresenta limites muito preocupantes em termos de buscas. A mesma busca realizada em dois computadores, um do lado do outro, no mesmo horário e dia, apresentou resultados diferentes. A única diferença entre as buscas foi o login de usuária.

Em síntese, quatorze artigos anglófonos e oito artigos brasileiros foram selecionados. A seguir, analisamos esses artigos para identificar sua literatura influente e a relevância atribuída às questões de gêneros pelos artigos de linguística.

\section{Os clássicos da linguística feminista e os temas de interesse pós- 2000}

Entendemos "clássicos" como a literatura (obra ou conjunto de obra) que sustenta o desenvolvimento de uma área, sendo referenciada com recorrência como base necessária para a constituição do campo, tendo ou não sofrido críticas desde sua publicação.

Nosso objetivo nesta seção é encontrar as obras de linguagem e gênero utilizadas como base para a linguística feminista, representada pelos artigos analisados. Num primeiro momento, trataremos de cada artigo separadamente, no que se refere aos estudos e autoras do campo de linguagem e gênero usados como base teórica.

Murnen (2000) se propõe a investigar se a linguagem usada por mulheres e homens para falarem sobre relações sexuais pode refletir e reproduzir a dominância masculina sobre a mulher por meio de termos que a diminuem e objetificam sexualmente. A autora faz breve alusão aos seguintes estudos sobre a interface linguagem, gênero e sexualidade: Janet Sanders e William Robinson (1979), Linda Carli (1990) e Crawford (1999).

Talbot (2000) investiga uma propaganda produzida por um canal de televisão inglês, o British Telecom, e exibida impressa na revista Radio Times em 1994, que aparentemente valoriza um estilo de conversa supostamente exclusivo das mulheres - a conversa fiada -, e incentiva os homens a falarem mais como as mulheres. Sob o viés da análise crítica do discurso, Talbot tem como apoio teórico três críticas sobre o livro de Tannen (1990) - uma de Senta Trömel-Plötz (1991), uma de Cameron (1992b) e uma de Alice Freed (1992). Uchida (1992), Cameron (1996) e produções da própria autora (TALBOT, 1997; 1998) também integram o artigo.

Em estudo sobre as relações entre bilinguismo, aquisição de segunda língua e gênero, Pavlenko (2001) encontra sua base teórica em estudos pós-estruturalistas sobre linguagem e gênero. Nesse sentido, a autora cita obras de: Butler (1990), Susan Gal (1991; 1995), Cameron (1992a), Eckert e McConnell-Ginet (1992), Tannen (1993), Don Kulick (1998a) e Talbot (1998).

Para investigar a ocorrência de argumentos heterossexistas em um treinamento para profissionais a respeito de como combater o heterossexismo e lidar com lésbicas e gays, Peel (2001) busca respaldo teórico em estudos de linguagem e gênero de Cameron (1998a,1998b), ${ }^{6}$ Mills (1998), e Sue Wilkinson e Kitzinger (1995).

Stapleton (2001) preocupa-se em mostrar como a abordagem de comunidade de prática pode ser adotada pela psicologia feminista. Para basear teoricamente esse posicionamento, evoca algumas produções sobre linguagem e gênero: Bucholtz (1999), Cameron (1997b; 1998a), Crawford (1995), Eckert e McConnell-Ginet (1992; 1995; 1999).

Os estudos a que Weatherall (2002) se alinha para fazer suas considerações e análises sobre a relevância do gênero para análise da conversação são de autoria de: Crawford (1995), Cameron (1998a), Elizabeth Stokoe (1998), e a própria autora (WEATHERALL, 2002).

Dentre os estudos que Mills (2003) usa para basear sua análise sobre as práticas de escoIha de sobrenome por mulheres casadas, estão os de Cameron (1998b; 1994; 1995b), Bucholtz (1999) e Janet Holmes e Miriam Meyerhoff (1999). Além desses, a autora também se baseia em suas produções (MILLS, 1995; 1998).

Philadelphoff-Puren (2004) demonstra que a recontextualização das recusas da vítima em um caso de estupro na Austrália pode interferir no resultado do julgamento do acusado. Recontextualização refere-se ao processo de ancoragem de textos em novos contextos; o processo

${ }^{6}$ Cameron (1998b) e Cameron (2005b) referem-se respectivamente à primeira e à segunda edição de The feminist critique of language: a reader. 
de recontextualização é um ato de controle, no qual operam questões de poder social (Richard BAUMAN; Charles BRIGGS, 1990). Os estudos de Butler (1997) e de Kitzinger e Hannah Frith (1999) fundamentam a análise de Philadelphoff-Puren.

A fim de indicar que o aspecto de identidade enfatizado nas pesquisas depende do método de análise escolhido pelas/os pesquisadoras/es e categorizar cinco princípios que representam como diferentes estudiosas/os abordam a identidade (emergência, posicionalidade, indexicalidade, relacionalidade e parcialidade), Bucholtz e Hall (2005) apoiam-se em West e Zimmerman (1983), Butler (1990), Goodwin (1990) e Cameron (1997a). Também são usadas como embasamento as publicações de Hall e O'Donavan (1996), Hall (1997), Hall e Anna Livia (1997), Eckert e McConnell-Ginet (1992; 2003) e Susan Ehrlich (2001).

Crawford e Fox (2007) examinam a proposta de uso dos termos sexo e gênero como uma reforma feminista da linguagem. Para isso, fazem uso das contribuições de Butler (1990), Cameron (1995b), Crawford (1995; 2001) e Anne Pauwels (1998).

Kitzinger e Rose Rickford (2007) centram-se em como a categoria de pertença de gênero é construída na fala-em-interação. As autoras não se estendem sobre um suporte teórico de produções precedentes, mas mencionam Butler (1990), Cameron (1997a), Kitzinger (2000; 2005a; 2005b), e Weatherall (2002). Já Lakoff (1975), Fishman (1983), Bent Preisler (1986) e Tannen (1990) são apresentados enquanto pesquisas teoricamente distantes.

Cameron (2006), Michelle Lazar (2005), Susan Speers (2005), Mills (2008) e Stokoe (2008) são os autoras de estudos de linguagem e gênero que fundamentam o artigo de Attenborough (2013), o qual consiste em analisar os processos de recontextualização usados nos textos midiáticos que lidam com o sexismo.

O objetivo de Cameron em seu artigo de 2015 é demonstrar que os desafios que a psicologia evolutiva impõe aos estudos feministas surgem mais dos trabalhos científicos populares do que da psicologia evolutiva em si. No artigo, são usados pela autora como base teórica: Kulick (1993) e Cameron (2006; 2007).

Weatherall (2015) avalia situações de sexismo na linguagem por meio da análise feminista da conversação. Os estudos de linguagem e gênero presentes no artigo são de: Butler (1990), Weatherall (1996; 2002), Kitzinger (2000; 2005a; 2005b; 2007) e Speers (2005).

Borba e Ostermann (2008) investigam como travestis que se prostituem em uma região urbana do Sul do Brasil manipulam o sistema de gênero gramatical. Para isso, a autora e o autor se embasam em Hall e O'Donovan (1996), Hall (1997), Kulick (1998a; 1998b), Jennifer Coates (1998), Cameron (2001), Bucholtz e Hall (2003; 2004; 2005), na tradução brasileira de Gender Trouble (BUTLER, 1990) em Butler (2003).

Ostermann (2008) faz comparações entre as práticas discursivas de profissionais que lidam com casos de violência contra as mulheres em uma unidade da Delegacia da Mulher e no centro feminista de intervenção contra esse tipo de violência. A autora coloca seu estudo junto àqueles que se preocupam com questões envolvidas em fazer gênero pela linguagem, cujas autoras são: Butler (1990), Eckert e McConnell-Ginet (1992; 1995; 1999), Gal (1995) e Stokoe (1998).

Butler (1990), Cameron (1992a; 1995b; 1998b), Ruth Wodak (1998) e Eckert e McConnell-Ginet (2003) são os autores das produções que apoiam o estudo que Débora Figueiredo (2009) desenvolve acerca das representações de corpo e identidade feminina em revistas para mulheres.

Debater discursos e identidades de gênero presentes no contexto social brasileiro é o propósito de Magalhães (2009). Bucholtz (1995) e Cameron (1995a; 1997a) configuram as produções utilizadas por Magalhães (2009) na construção desse debate entre os discursos sobre gênero.

Sell e Ostermann (2009) estão preocupadas em entender como identidades de gênero são negociadas na fala-em-interação entre uma psicóloga e seus pacientes que buscam uma vasectomia em um posto de atendimento do Sistema Único de Saúde. As autoras, que adotam uma perspectiva pós-estruturalista, apoiam-se em publicações de: Butler (1990; 1993), Bucholtz e Hall $(2003 ; 2004 ; 2005)$ e Cameron (2005a).

Pinto e Badan (2012) discutem a respeito das articulações entre teoria e descrição linguística com identidades feministas. Assim, as bases teóricas das autoras se encontram em: Cameron (1992a; 1998a), Cameron et al. (1993) e Carmen Caldas-Coulthard (2007).

Os estudos de linguagem e gênero que orientam a pesquisa de Sell (2012) sobre as identidades de gênero e maternidade negociadas na fala-em-interação entre uma psicóloga e suas e seus pacientes que buscam uma laqueadura e vasectomia são as de Butler (1990), Eckert e McConnell-Ginet (1992), Bucholtz e Hall (2005).

Andrade, Carvalho e Zambrano (2017) voltam-se às representações de gênero em verbetes sobre profissões em dicionários de alemão, espanhol e português. Apoiando teoricamente esse estudo estão Butler (1990; 1993), Lazar (2005; 2007), e Mills (2008). 
Embora apenas quatro artigos mencionem as produções de Eckert e McConnell-Ginet, as autoras se destacam em relação à quantidade de trabalhos citados nesses quatro artigos. Stapleton (2001) e Ostermann (2008) trazem como base Eckert e McConnell-Ginet (1992; 1995; 1999). Já Bucholtz e Hall (2005) apoiam-se em Eckert e McConnell-Ginet (1992; 2003) e Figueiredo (2009), e em Eckert e McConnell-Ginet (2003).

A obra mais citada nos 22 artigos analisados é de autoria de Butler (1990). Gender Trouble: feminism and the subversion of identity influenciou onze artigos, cinco anglófonos e seis brasileiros. Apenas dois artigos se valem de outras obras de Butler: Philadelphoff-Puren (2004) cita apenas Butler (1997) e Sell e Ostermann (2009) citam Butler (1990; 1993).

No que se refere ao conjunto de produções mais citadas, o crédito fica com a linguista britânica Cameron. Quinze artigos fazem uso das produções de Cameron na construção de suas bases teóricas. Nenhuma obra da autora se consagra como a mais citada, mas se destacam "Feminism and linguistic theory", "Verbal hygiene", "Gender, language, and discourse: a review essay", "Performing gender identity: Young man's talk and the construction of heterossexual masculinity" e "The feminist critique of language: a reader" (CAMERON, 1992a; 1995b; 1998a; 1997a; 2005b [1998b]).

\section{A relevância do gênero para a linguística feminista}

Os debates entre análise da conversa e análise crítica do discurso sobre a relevância do gênero na linguagem e nas decisões políticas na análise linguística e a hipótese de consolidação do campo da linguística feminista nos levaram a pensar sobre como questões de gênero são avaliadas nas pesquisas sobre linguagem.

Agora, voltamo-nos ao posicionamento do artigo em relação à discussão sobre a relevância do gênero para a pesquisa em linguagem. Nesta parte da análise, oferecemos às/ aos leitoras/es observações sobre as articulações gênero/linguagem/pesquisa encontradas nos artigos selecionados, ilustrando com partes e especificidades dos artigos quando pertinente.

Ao longo desta pesquisa, percebemos que, ainda que todos os artigos discutam gênero e linguagem, são poucos os artigos que abordam explicitamente a relevância do gênero para os estudos da linguagem, ou seja, a maioria dos artigos simplesmente assume como óbvia a relevância da categoria gênero para suas análises. Por exemplo, para Sell (2012), quando se assume "a linguagem como prática corporeificada, não se pode mais conceber as identidades de gênero como existentes fora do discurso" (SELL, 2012, p. 155). Estudar a linguagem pelo viés de gênero se justifica, portanto, por não ser possível construir identidades de gênero em outro enquadramento que não a linguagem; não há necessidade de argumentar sobre a importância de que gênero entre na equação teórico-analítica.

De forma semelhante, Borba e Ostermann (2008, p. 410) apontam que um estudo das intersecções entre gênero e linguagem acontece para "entender como os/as transgêneros utilizam a linguagem para se alocar nas e/ou transcender as fronteiras dos gêneros". Figueiredo (2009, p. 739) também faz considerações sobre a importância dessas intersecções:

A construção identitária, incluindo a construção do corpo, se torna possivel através das narrativas e representações que caracterizam cada sociedade, com base nas quais os sujeitos constroem narrativas individuais minimamente coerentes. E podemos afirmar que é através dos textos que circulam em suas comunidades que os sujeitos sociais se familiarizam com os modelos narrativos e representacionais presentes na cultura e constroem suas identidades através de representações. Do ponto de vista dos estudos de gênero e dos estudos identitários, os textos, e as representações culturais que eles constroem, são espaços privilegiados para explorar as possibilidades abertas para os indivíduos em sua constituição como sujeitos de gênero.

Enquanto procurávamos entender o olhar das pesquisas em linguagem e gênero sobre a interface gênero, especificamente, encontramos em alguns artigos considerações a respeito da linguagem. Por estar interessada em examinar questões de gênero e sexualidade por meio da linguagem, Murnen (2000) procura justificar o porquê de a linguagem estar no cerne de seu estudo de psicologia feminista: "usamos a linguagem para fazer sentido do mundo e compartilhar nossas percepções com outras pessoas. [...] Por isso, a linguagem um importante mecanismo envolvido tanto na descrição quanto na criação das construções sociais de eventos" (MURNEN, 2000 , p. 319). Em outro artigo de psicologia feminista, Peel (2001), de forma breve, diz que, após a virada linguística nas ciências sociais, pesquisas feministas passaram a se preocupar com a linguagem sexista e a maneira como ela produz e reflete desigualdades "sexuais". Novamente, justifica-se o item "linguagem" no duo linguagem-gênero.

Embora não se centre propriamente na pertinência do gênero para os estudos da linguagem, ou da linguagem para os estudos de gênero, Stapleton (2001) afirma que os estudos da época estavam interessados em como a linguagem "reflete, cria, mantém ou desafia sistemas e ideologias patriarcais" (p. 460). Interessada nos impactos benéficos para seu próprio campo 
de estudos, a psicologia feminista, a autora se estende acerca das qualidades positivas das implicações e características da abordagem de comunidade de práticas para a pesquisa em psicologia feminista.

Dos artigos analisados, o único que de fato trata do papel do gênero para a pesquisa parece ser o de Weatherall (2000), da análise da conversa. A autora dedica algumas páginas de seu artigo para discutir acerca dessa questão. Na medida em que se preocupa tanto como é realmente relevante nas interações analisadas, quanto em manter uma postura crítica como pesquisadora feminista, Weatherall entende que "uma análise de gênero é potencialmente relevante para qualquer interação porque gênero é uma categoria social que se infiltra" e que é papel do/da pesquisador/a demonstrar "como e que participantes [da interação] mostram sua orientação quanto à onirelevância do gênero" (WEATHERALL, 2002, p. 779).

Uma vez que a relevância do gênero e outras questões sobre identidades e tratamento do contexto são extensivamente abordadas em alguns estudos que tratam de conteúdo teórico-metodológico (SCHEGLOFF, 1997; 1999; Jan BLOMMAERT, 2001; BUCHOLTZ, 2003), seria de se esperar que as autoras e autores de linguagem e gênero se estendessem sobre a importância de alguma dessas interfaces das interações em seus artigos. Parece, todavia, que o papel do gênero na linguagem torna-se óbvio, taken for granted, para o campo no cenário anglófono, visto que apenas um quinto dos artigos anglófonos (łodos da mesma época, início dos anos 2000) investe em expor as razões para analisar aspectos de linguagem na constituição do gênero e todos são da psicologia feminista, nenhum da linguística.

Ainda que os estudos sobre gênero e linguagem pós-2000 realizados em instituições brasileiras não sejam exatamente pioneiros, já que temos estudos como os de Ostermann (1994; 1995) e Figueiredo (1997; 1998a; 1998b) anteriores ao novo século, ${ }^{7}$ essa divergência entre os cenários pode ser creditada à circunstância desse tipo de pesquisa no Brasil ter apenas três décadas, em vez das cinco décadas na produção anglófona. Nos países anglófonos, de modo oposto, a falta de uma colocação quanto à relevância de gênero pode indicar um campo de estudos mais estabelecido em suas bases teóricas, assim como corroborar Weatherall, quando ela diz que "para todas as feministas gênero é, teoricamente, relevante para qualquer interação" (2002, p. 768).

\section{Conclusōes}

Os portais buscadores impuseram limites a esta pesquisa. O Portal de Periódicos da Capes apresentou muitos resultados repetidos ou fora dos critérios, porque listava tanto artigos completos quanto resumos, além de listar resenhas, e acabava buscando os termos fora do campo de palavras-chave, mesmo que tenhamos selecionado somente este campo. O viés de usuária do Google Scholar, por sua vez, torna difícil confiar nas suas buscas como base para um levantamento bibliográfico consistente, já que o próprio buscador seleciona o que cada usuária deve encontrar, comprometendo a replicabilidade da pesquisa.

Ainda assim, esta pesquisa foi rigorosa na aplicação dos critérios e confiamos que seus resultados mostram, se não um cenário definitivo, um indicativo dos movimentos teóricos e metodológicos do campo da linguística feminista contemporânea. Esperamos que possa servir como mais uma possibilidade de apresentar, debater e ampliar este campo no Brasil.

Os estudos feministas do campo de linguagem e gênero têm se mostrado um cenário produtivo para colocar em foco questões sociais e culturais de sexo e gênero que se constroem pela linguagem (BUTLER, 1997). Nesse sentido, e tendo em vista mais de meio século de produÇão de conhecimento científico dos estudos de linguagem e gênero, buscamos entender alguns aspectos do funcionamento contemporâneo do campo. Assim, uma pesquisa bibliográfica pelos artigos anglófonos e brasileiros de linguística feminista produzidos entre 2000 e 2017 e suas bases bibliográficas nos apresentou alguns índices de consolidação do campo.

Considerando a pergunta sobre quais são as obras clássicas para a linguística feminista que influenciam o campo a partir dos anos 2000, podemos dizer que Butler e Cameron são as autoras que se distinguem como influências para os artigos. Porém, ao passo que das obras de Butler apenas Gender Trouble (1990) pode ser tomada como uma obra clássica, várias produções de Cameron têm peso sobre os artigos analisados, de forma que concluímos que o conjunto da obra da linguista britânica se valida como clássico.

Haja vista a segunda pergunta sobre a preocupação em estipular a relevância do gênero para se estudar linguagem, concluímos que, de forma geral, nos artigos analisados não

\footnotetext{
${ }^{7}$ A brasileira Caldas-Coulthard é, sem dúvida, uma das pessoas mais influentes para uma geração de mulheres linguistas feministas brasileiras; suas publicações foram fundamentais para estimular a produção de pesquisa nacional no campo, e sua longa estadia como professora e pesquisadora na Inglaterra (de 1996 a 2012) não cessou sua importância para nossos trabalhos. No entanto, sua produção foi publicada predominantemente fora do Brasil, não atendendo aqui aos nossos critérios para "produção brasileira". É o caso, por exemplo, de Caldas-Coulthard (2007) que, mesmo tendo sido publicado em português em revista internacional, foi publicado quando a autora ainda era professora da University of Birmingham, Reino Unido.
} 
há um empenho por parte das autoras e autores em discutir acerca da relevância de gênero para a pesquisa em linguagem. Com exceção dos artigos que procuram abordar aspectos da fala-em-interação para demonstrar que gênero é relevante na conversação, consideramos que os estudos de linguagem e gênero têm a importância de gênero dada como óbvia.

Entendemos que essa falta de discussão metateórica pode ser um índice da consolidação do campo, em que a relevância de gênero já parece estar configurada como um pressuposto teórico. Isso se verifica nas produções anglófonas, dentre as quais apenas um quinto, do início dos anos 2000 , procura justificar a interface gênero e linguagem da pesquisa, com ênfase em linguagem e não em gênero. Contrariamente, o relativo maior empenho das produções brasileiras em justificar uma análise de linguagem e gênero parece surgir do caráter ainda recente dessas pesquisas no Brasil.

\section{Referências}

ANDRADE, Giselly; CARVALHO, Gislene; ZAMBRANO, Romana. "A representação do gênero em dicionários monolíngues dos idiomas alemão, espanhol e português: uma análise crítica feminista de verbetes referentes às profissões". Calidoscópio, São Leopoldo, v. 15, n. 3, p. 433 442, set./dez. 2017.

ATTENBOROUGH, Frederick. "Jokes, pranks, blondes and banter: recontextualising sexism in the British print press". Journal of Gender Studies, v. 23, n. 2, p. 137-154, 2013 [on-line]. Disponível em https://doi.org/10.1080/09589236.2013.774269. Acesso em 11/07/2018.

BAUMAN, Richard; BRIGGS, Charles. "Poetics and performance as critical perspectives on language and social life". Annual Review of Anthropology, v. 19, p. 59-88, out. 1990.

BLOMMAERT, Jan. "Context is/as critique". Critique of anthropology, v. 21, n. 1, p. 13-32, 2001 [online]. Disponível em https://doi.org/10.1177/0308275X0102100102. Acesso em 02/03/2018.

BORBA, Rodrigo; OSTERMANN, Ana Cristina. "Gênero ilimitado: a construção discursiva da identidade travesti através da manipulação do sistema de gênero gramatical". Revista Estudos Feministas, Florianópolis, v. 16, n. 2, p. 409-432, maio/ago. 2008.

BUCHOLTZ, Mary. “'Why be normal?': language and identity practices in a community of nerd girls". Language in Society, v. 28, n. 2, p. 203-223, 1999 [on-line]. ISSN 1469-8013. Disponível em doi.org/10.1017/S0047404599002043.

BUCHOLTZ, Mary. "Editor's introduction". In: LAKOFF, Robin. Language and Women's Place. 2. ed. Nova York: Oxford University Press, 2004. p. 3-14.

BUCHOLTZ, Mary. "From mulatta to mestiza: passing and the linguistic reshaping of ethnic identity". In: HALL, Kira; BUCHOLTZ, Mary (Eds.). Gender articulated: language and the socially constructed self. Londres: Routledge, 1995. p. 351-373.

BUCHOLTZ, Mary. "Theories of discourse as theories of gender: discourse analysis in language and gender studies". In: HOLMES, Janet; MEYERHOFF, Miriam (Eds.). The handbook of language and gender. Malden: Blackwell Publishing, 2003. p. 43-68.

BUCHOLTZ, Mary; HALL, Kira. "Identity and interaction: a sociocultural linguistic approach". Discourse Studies, v. 7, n. 4-5, p. 585-614, 2005 [on-line]. Disponível em https://doi. org/10.1177/1461445605054407. Acesso em 20/07/2018.

BUCHOLTZ, Mary; HALL, Kira. "Language and identity". In: DURANTI, Alessandro. (Ed.). A companion to linguistic anthropology. Oxford: Blackwell, 2003. p. 268-294.

BUCHOLTZ, Mary; HALL, Kira. "Theorizing identity in language and sexuality research". Language in Society, v. 33, n. 4, p. 469-515, 2004 [on-line]. Disponível em doi.org/10.1017/S0047404504334020.

BUTLER, Judith. "Fundamentos contingentes: o feminismo e a questão do 'pós-modernismo'". Cadernos pagu, Campinas, v. 11, p. 11-42, 1998.

BUTLER, Judith. Excitable speech. Nova York: Routledge, 1997.

BUTLER, Judith. Gender Trouble: feminism and the subversion of identity. Nova York: Routledge, 1990.

BUTLER, Judith. Problemas de gênero. Civilização Brasileira: Rio de Janeiro, 2003. 
CALDAS-COULTHARD, Carmen R. "Caro colega: exclusão linguística e invisibilidade". Discurso \& Sociedad, v. 1, n. 2, p. 230-246, 2007 [on-line]. Disponível em http://dissoc.org/ediciones/v01n02/ DS1(2)Caldas-Coulthard.pdf. ISSN 1887-4606. Acesso em 28/01/2018.

CAMERON, Deborah et al. "Ethics, advocacy and empowerment: issues of method in researching language". Language and communication, v. 13, n. 2, p. 81-94, 1993 [on-line]. DOI doi. org/10.1016/0271-5309(93)90001-4.

CAMERON, Deborah. "Evolution, language, and the battle of the sexes". Australian Feminist Studies, v. 30, n. 86, p. 351-358, 2015 [on-line]. ISSN 1465-3303. Disponível em https://dol.org/10.1 080/08164649.2016.1148097. Acesso em 16/07/2018.

CAMERON, Deborah. "Gender, language, and discourse: a review essay". Signs, Boston, v. 23, n. 4, p. 945-973, 1998a.

CAMERON, Deborah. "Language, gender, and sexuality: current issues and new directions". Applied Linguistics, v. 26, n. 4, p. 482-502, 2005a [on-line]. Disponível em doi.org/10.1093/applin/ ami027.

CAMERON, Deborah. "Performing gender identity: young men's talk and the construction of heterosexual masculinity". In: JOHNSON, Sally; MEINHOF, Ulrike (Eds.). Language and masculinity, Oxford: Blackwell, 1997a. p. 47-64.

CAMERON, Deborah. "Rethinking gender and language studies: some issues for the 1990s". In: MILLS, Sara (Ed.). Language and gender: interdisciplinary perspectives. Londres: Longman, 1995a. p. 31-44.

CAMERON, Deborah. "Review of Deborah Tannen". Feminism and Psychology, v. 2, n. 3, p. 465489, $1992 \mathrm{~b}$.

CAMERON, Deborah. "The language-gender interface: challenging co-optation". In: BERGVALL, Victoria; BING, Janet; FREED, Alice (Orgs.). Rethinking gender and language research: theory and practice. Londres: Longman, 1996. p. 31-53.

CAMERON, Deborah. "Theoretical debates in feminist linguistics: questions of sex and gender". In: WODAK, Ruth (Ed.). Gender and discourse. Londres: Sage, 1997b. p. 21-36.

CAMERON, Deborah. "Why is language a feminist issue?". In: CAMERON, Deborah (Ed.). The feminist critique of language: a reader. 2. ed. Abingdon: Routledge, 2005b [1998b]. p. 1-21.

CAMERON, Deborah. "Words, words, words: the power of language". In: DUNANT, Sarah (Ed.). The war of the words: the political correctness debate. Londres: Virago, 1994. p. 15-34.

CAMERON, Deborah. Feminism and linguistic theory. Londres: McMillan, 1992a.

CAMERON, Deborah. Language and sexual politics. Londres: Routledge, 2006.

CAMERON, Deborah. The feminist critique of language: a reader. 1. ed. Abingdon: Routledge, 1998b.

CAMERON, Deborah. The myth of mars and venus: do men and women really speak different languages? Oxford: Oxford University Press, 2007.

CAMERON, Deborah. Verbal hygiene. Londres: Routledge, 1995b.

CAMERON, Deborah. Working with spoken discourse. Londres: Sage, 2001.

CARLI, Linda. "Gender, language and influence". Journal of Personality and Social Psychology, v. 59, p. 941-951, 1990 [on-line]. Disponível em 10.1037/0022-3514.59.5.941.

COATES, Jennifer. “'Thank God I'm a woman': the construction of differing femininities”. In: CAMERON, Deborah (Ed.). The feminist critique of language: a reader. 2. ed. Abingdon: Routledge, 2005. p. 297-320

CRAWFORD, Mary. "Gender and language". In: UNGER, Rhoda (Ed.). Handbook of the psychology of women and gender. Nova York: Wiley, 2001. p. 245-255. 
CRAWFORD, Mary. "Only joking: Humor and sexuality". In: TRATIS, Cheryl; WHITE, Jacquelyn (Eds.). Sexuality, society, and feminism. Washington, DC: American Psychological Association, 1999. p. 213-236.

CRAWFORD, Mary. Talking difference: on gender and language. Londres: Sage, 1995.

CRAWFORD, Mary; FOX, Annie. "From sex to gender and back again: co-optation of a feminist language reform". Feminism and Psychology, v. 7, n. 4, p. 481-486, 2007 [on-line]. Disponível em https://doi.org/10.1177/0959353507084333. Acesso em 17/07/2018. ISSN 1461-7161.

ECKERT, Penelope; MCCONNELL-GINET, Sally. "Constructing meaning, constructing selves: snapshots of language, gender and class from Belten High". In: HALL, Kira;BUCHOLTZ, Mary (Eds.). Gender articulated: language and the socially constructed self. Londres: Routledge, 1995. p. 469507.

ECKERT, Penelope; MCCONNELL-GINET, Sally. "New generalizations and explanations in language and gender research". Language in Society, v. 28, n. 2, p. 185-201, 1999 [on-line]. Disponível em doi.org/10.1017/S0047404599002031.

ECKERT, Penelope; MCCONNELL-GINET, Sally. Language and gender. Cambridge: Cambridge University Press, 2003

ECKERT, Penelope; MCCONNELL-GINET, Sally. "Think practically and look locally: language and gender as community-based practice". Annual Review of Anthropology, v. 21, p. 461-490, out. 1992.

EHRLICH, Susan. Representing rape: language and sexual consent. Nova York: Routledge, 2001.

FIGUEIREDO, Débora. "Discurso, gênero e identidade: Uma análise crítica de sentenças jurídicas em casos de estupro". Revista Ciências da Saúde, Florianópolis, v. 17, n. 1, p. 214-232, 1998a.

FIGUEIREDO, Débora. "Gênero e poder no discurso jurídico". Revista de Clências Humanas, Florianópolis, v. 15, n. 21, p. 37-52, 1997.

FIGUEIREDO, Débora. "Identities and gender in the discourse of rape trials". Ilha do Desterro, Florianópolis, UFSC, v. 7, n. 2, p. 85-102, 1998b.

FIGUEIREDO, Débora. "Linguagem e gênero social: contribuições da análise crítica do discurso e da linguística sistêmico-funcional”. D.E.L.T.A., São Paulo, v. 25, número especial, p. 732-753, 2009.

FISHMAN, Pamela. "Interaction: the work women do". In: THORNE, Barrie; KRAMARAE, Cheris HENLEY, Nancy (Eds.). Language, gender and society. Cambridge: Newbury House, 1983. p. 89-101.

FREED, Alice. "We understand perfectly: a critique of Tannen's view of cross-sex communication". In: HALL, Kira; BUCHOLTZ, Mary; MOONWOMON, Birch (Eds.). Locating power: proceedings of the Second Berkeley Women and Language Conference. Berkeley: Berkeley Women and Language Group, 1992. p. 144-152.

GAL, Susan. "Between speech and silence: the problematics of research on language and gender". In: LEONARDO, Micaela di (Ed.). Gender at the crossroads of knowledge. Berkeley: University of California Press, 1991. p. 175-203.

GAL, Susan. "Language, gender, and power: an anthropological view". In: HALL, Kira; BUCHOLTZ, Mary; MOONWOMON, Birch (Eds.). Locating power: proceedings of the Second Berkeley Women and Language Conference. Berkeley: Berkeley Women and Language Group, 1995. p. 153-161.

GALLOP, Jane. "Women" in Spurs and nineties feminism". In: FEDER, Ellen K. et al. (Eds.). Derrida and feminism: recasting the question of woman. Nova York: Routledge, 1997. p. 7-19.

GOODWIN, Marjorie H. He-said-she-said: talk as social organization among black children. Bloomington: Indiana University Press, 1990.

GOUGH, Val; TALBOT, Mary. "'Guilt over games boys play': coherence as a focus for examining the constitution of heterosexual subjectivity on a problem page". In: CALDAS-COULTHARD, Carmen R.; COULTHARD, Malcolm (Eds.). Texts and practices: reading in critical discourse analysis. Londres: Routledge, 1996. p. 215-230. 
HALL, Kira. "'Go suck your husband's sugarcane': hijras and use of sexual insult”. In: LIVIA, Anna; HALL, Kira (Eds.). Queerly phrased: language, gender and sexuality. Nova York: Oxford University Press, 1997. p. 430-460.

HALL, Kira; LIVIA, Anna. “'It's a girl!': bringing performativity back to linguistics”. In: LIVIA, Anna; HALL, Kira (Ed.). Queerly phrased: language, gender and sexuality. Nova York: Oxford University Press, 1997. p. 3-18.

HALL, Kira; O'DONAVAN, Veronica. "Shifting gender positions among Hindi-speaking hijras". In: BERGVALL, Victoria; BING, Janet; FREED, Alice (Eds.). Rethinking gender and language research: theory and practice. Londres: Longman, 1996. p. 228-266.

HOLMES, Janet; MEYERHOFF, Miriam. "The community of practice: theories and methodologies in language and gender research". Language in Society, v. 28, n. 2, p. 178-183, 1999 [on-line]. Disponível em doi.org/10.1017/S004740459900202X.

JESPERSEN, Otto. "The woman". In: CAMERON, Deborah (Ed.). The feminist critique of language: a reader. 2. ed. Abingdon: Routledge, 2005. p. 225-241.

KITZINGER, Celia. "Doing feminist conversational analysis". Feminism and Psychology, v. 10, n. 2, p. 163-193, 2000. DOI dol.org/10.1177/0959353500010002001.

KITZINGER, Celia. "Heteronormativity in action: reproducing the heterosexual nuclear family in after-hours medical calls". Social Problem, v. 52, n. 4, p. 477-498, nov. 2005a. DOI doi.org/10.1525/ sp. 2005.52.4.477.

KITZINGER, Celia. "Speaking as heterosexual: (how) does sexuality matter for talk-in-interaction?". Research on Language and Social Interaction, v. 38, n. 1, p. 221-265, 2005b.

KITZINGER, Celia. "Is 'woman' always relevantly gendered?". Gender and language, v. 1, n. 1, p. 39-50, 2007 [on-line]. Disponível em 10.1558/genl.2007.1.1.39.

KITZINGER, Celia; FRITH, Hannah. "Just say no? The use of conversational analysis in developing a feminist perspective on sexual refusal". Discourse and Society, v. 10, n. 3, p. 293-316, 1999. Disponível em doi.org/10.1177/0957926599010003002.

KITZINGER, Celia; RICKFORD, Rose. "Becoming a bloke: the construction of gender in interaction". Feminism and Psychology, v. 17, n. 2, p. 214-223, 2007 [on-line]. ISSN 1461-7161. Disponível em https://doi.org/10.1177/0959353507076554. Acesso em 23/07/2018.

KULICK, Don. "Anger, gender, language shift, and the politics of revelation in a Papua New Guinean village". In: SCHIEFFELIN, Bambi; WOOLARD, Kathryn.; KROSKRITY, Paul (Eds.). Language ideologies: practice and theory. Nova York: Oxford University Press, 1998a. p. 87-102.

KULICK, Don. "Speaking as a woman: structure and gender in domestic arguments in a Papua New Guinea village". Cultural Anthropology, v. 8, n. 4, p. 510-541, nov. 1993.

KULICK, Don. Travesti: sex, gender, and culture among Brazilian transgendered prostitutes. Chicago: Chicago University press, 1998b.

LAKOFF, Robin. Language and Women's Place. 2. ed. Nova York: Oxford University Press, 2004.

LAKOFF, Robin. Language and Women's Place. Nova York: Harper and Now, 1975.

LAZAR, Michelle. "Feminist critical discourse analysis: articulating a feminist discourse praxis". Critical Discourse Studies, v. 4, n. 2, p. 141-164, 2007 [on-line]. DOI dol.org/10.1080/17405900701464816.

LAZAR, Michelle. Feminist critical discourse analysis. Basingstoke: Palgrave, 2005.

MAGALHÃES, Izabel. "Gênero e discurso no Brasil". Discurso \& Sociedad, v. 3, n. 4, p. 714-737, 2009. Disponível em http://www.dissoc.org/ediciones/v03n04/DS3(4)Magalhaes.pdf. ISSN 18874606. Acesso em 05/02/2018.

MATHIEU, Nicole-Claude. "Sexual, sexed and sex-Class identities: three ways of conceptualising the relationship between sex and gender". In: LEONARD, Diana; ADKINS, Lisa (Eds.). Sex in question: French materialist feminism. Londres: Taylor and Francis, 2005. p. 43-72. 
McELHINNY, Bonnie. "Theorizing gender in sociolinguistics and linguistic anthropology". In: HOLMES, Janet; MEYERHOFF, Miriam (Eds.). The handbook of language and gender. Malden: Blackwell, 2003. p. 21-42.

MILLS, Sara. "Caught between sexism, anti-sexism and 'political correctness': feminist women's negotiations with naming practices". Discourse and Society, v. 14, n. 1, p. 87-110, 2003. ISSN 1460-3624. Disponível em https://dol.org/10.1177/0957926503014001931. Acesso em 22/07/2018.

MILLS, Sara. "Post feminism text analysis". Language and Literature, v. 7, n. 3, p. 235-253, 1998.

MILLS, Sara. Feminist stylistics. Londres: Routledge, 1995.

MILLS, Sara. Language and sexism. Cambridge: Cambridge University Press, 2008.

MURNEN, Sarah. "Gender and the use of sexually degrading language". Psychology of Women Quaterly, v. 24, p. 319-327, 2000 [on-line]. ISSN 1471-6402. Disponível em https://doi. org/10.1111/j.1471-6402.2000.tb00214.x. Acesso em 18/06/2018.

OSTERMANN, Ana Cristina. "Análise da conversa (aplicada) como uma abordagem para o estudo de linguagem e gênero: o caso dos atendimentos a mulheres em situação de violência no Brasil". Athenea Digital, n. 14, p. 245-266, 2008. Disponível em https://atheneadigital.net/article/ viewFile/n14-ostermann/537-pdf-pt. ISSN 1578-8946. Acesso em 18/07/2018.

OSTERMANN, Ana Cristina. "Bonita de doer: análise crítica do discurso em revistas para meninas adolescentes". The Especialist, São Paulo, v. 15, n. 1-2, p. 151-162, 1995.

OSTERMANN, Ana Cristina. "Male's Dominance in Conversational Interactions". Verso e Reverso, São Leopoldo, v. 15, n. 1, p. 127-133, 1994.

OSTERMANN, Ana Cristina; FONTANA, Beatriz. "Linguagem. Gênero. Sexualidade: uma introdução". In: OSTERMANN, Ana Cristina; FONTANA, Beatriz (Orgs.). Linguagem, gênero, sexualidade: clássicos traduzidos. São Paulo: Parábola, 2010. p. 9-12.

PAUWELS, Anne. Women changing language. Nova York: Addison Wesley Longman, 1998.

PAVLENKO, Aneta. "Bilingualism, gender and ideology". The International Journal of Bilingualism, v. 5, n. 2, p. 117-151, 2001 [on-line]. ISSN 1756-6878. Disponível em https://doi.org/10.1177/136700 69010050020101. Acesso em 19/07/2018.

PEEL, Elizabeth. "Mundane heterosexism: understanding incidents of the everyday". Women's Studies International Forum, v. 24, n. 5, p. 541-554, 2001. Disponível em https://www.sciencedirect. com/science/article/abs/pii/S0277539501001947. Acesso em 20/07/2018.

PHILADELPHOFF-PUREN, Nina. "The mark of refusal: sexual violence and the politics of recontextualization". Feminist Theory, v. 5, n. 3, p. 243-156, 2004 [on-line]. ISSN 1741-2773. Disponível em https://doi.org/10.1177/1464700104046975. Acesso em 25/06/2018.

PINTO, Joana Plaza; BADAN, Suzana C. "Feminismo e as identidades no cerne dos princípios de pesquisa". Calidoscópio, São Leopoldo, v. 10, n. 2, p. 133-139, 2012.

PISCITELLI, Adriana G. "Recriando a (categoria) mulher?". In: ALGRANTI, Leila (Org.). A prática feminista e o conceito de gênero. Campinas: IFCH/Unicamp, 2002. p. 7-42.

PREISLER, Bent. Linguistic sex roles in conversation. Amsterdam: Mouton de Gruyter, 1986.

RUBIN, Gayle. "The traffic in women: notes on the "political economy" of sex". In: REITER, Rayna. Toward an Anthropology of Women. Nova York: Monthly Review Press, 1975. p. 157-210.

SANDERS, Janet; ROBINSON, William. "Talking and not talking about sex: male and female vocabularies". Journal of Communication, v. 29, n. 2, p. 22-30, 1979.

SCHEGLOFF, Emanuel. "Naivete vs. sophistication or discipline vs. self-indulgence: a rejoinder to Billig". Discourse and Society, v. 10, n. 4, p. 577-582, 1999 [on-line]. ISSN 1460-3624. Disponível em https://doi.org/10.1177/0957926599010004008. Acesso em 22/04/2018.

SCHEGLOFF, Emanuel. "Whose text? Whose context?". Discourse and Society, v. 8, n. 2, p. 165-187, 1997. ISSN 1460-3624. Disponível em https://doi.org/10.1177/0957926597008002002. Acesso em $22 / 04 / 2018$. 
SELL, Mariléia. “'Minha mãe ficou amarga': expectativas de performances de maternidade negociadas na fala-em-interação". Revista Estudos Feministas, Florianópolis, v. 20, n. 1, p. 153172, 2012.

SELL, Mariléia; OSTERMANN, Ana Cristina. "Análise de Categorias de Pertença (ACP) em estudos de linguagem e gênero: a (des)construção discursiva do homogêneo masculino". Alfa, Araraquara, v. 53, p. 11-34, 2009.

SPEERS, Susan. Gender talk. Londres: Routledge, 2005.

STAPLETON, Karyn. "Constructing a feminist identity: discourse and the community of practice". Feminism and Psychology, v. 11, n. 4, p. 459-491, 2001 [on-line]. ISSN 1461-7161. Disponível em https://doi.org/10.1177/095935350101 1004003. Acesso em 13/07/2018.

STOCKILL, Clare; KITZINGER, Celia. "Gendered people: how linguistically non-gender terms can have gendered interactional relevance". Feminism and Psychology, v. 17, n. 2, p. 224-236, 2007 [on-line]. ISSN 1461-7161. Disponível em https://doi.org/10.1177/0959353507076555. Acesso em 23/07/2018.

STOKOE, Elizabeth. "Categories and sequences: formulating gender in talk-in-interaction". In: HARRINGTON, Kate et al. (Eds.). Gender and language research methodologies. Basingstoke: Palgrave, 2008.

STOKOE, Elizabeth. "Talking about gender: the conversational construction of gender categories in academic discourse". Discourse and Society, v. 9, n. 2, p. 217-240, abr. 1998. Disponível em doi.org/10.1177/0957926598009002005.

TALBOT, Mary. "'Randy fish boss branded a stinker': coherence and the construction of masculinities in a British tabloid newspaper". In: JOHNSON, Sally; MEINHOF, Ulrike (Eds.). Language and masculinity, Oxford: Blackwell, 1997. p. 173-187.

TALBOT, Mary. "A synthetic sisterhood: false friends in a teenage magazine". In: HALL, Kira; BUCHOLTZ, Mary (Eds.). Gender articulated: language and the socially constructed self. Londres: Routledge, 1995. p. 143-165.

TALBOT, Mary. "It's good to talk? The undermining of feminism in a British Telecom advertisement". Journal of Sociolinguistics, v. 4, n. 1, p. 108-119, 2000 [on-line]. ISSN 1467-9841. Disponível em https://doi.org/10.1111/1467-9481.00105. Acesso em 21/04/2018.

TALBOT, Mary. Language and gender: an introduction. Cambridge: Polity, 1998.

TANNEN, Deborah. "The display of (gendered) identities in talk at work". In: BUCHOLTZ, Mary; LIANG, A. C.; SUTTON, Laurel (Eds.). Reinventing identities: the gendered self in discourse. Nova York: Oxford University Press, 1999. p. 221-240.

TANNEN, Deborah. "The relativity of linguistic strategies: rethinking power and solidarity in gender and dominance". In: TANNEN, Deborah (Ed.). Gender and conversational interaction. Oxford: Oxford University Press, 1993. p. 165-188.

TANNEN, Deborah. You just don't understand: women and men in conversation. Nova York: William Morrow, 1990.

TRÖMEL-PLÖTZ, Senta. "Review essay: Selling the apolitical". Discourse and Society, v. 2, n. 4, p. 498-502, 1991.

UCHIDA, Aki. "When difference is dominance: a critique of the 'anti-power based' cultural approach to sex differences". Language in Society, v. 21, n. 4, p. 547-568, 1992.

UCHIDA, Aki. 'When 'difference' is 'dominance': a critique of the 'anti-power-based' cultural approach to sex differences". In: CAMERON, Deborah (Ed.). The feminist critique of language: a reader. 2. ed. Abingdon: Routledge, 2005. p. 280-292.

WEATHERALL, Ann. "Gender relevance in talk-in-interaction and discourse". Discourse and Society, v. 11, n. 2, p. 286-288, 2000 [on-line]. ISSN 1460-3624. Disponível em https://doi. org/10.1177\%2F0957926500011002012. Acesso em 23/04/2018. 
WEATHERALL, Ann. "Language about men and women: an example from popular culture". Journal of Language and Social Psychology, v. 15, n. 1, p. 59-75, mar. 1996. Disponível em doi. org/10.1177/0261927X960151004.

WEATHERALL, Ann. "Sexism in language and talk-in-interaction". Journal of Language and Social Psychology, v. 34, n. 4, p. 410-426, 2015 [on-line]. ISSN 1552-6526. Disponível em https://doi. org/10.1177/0261927X15586574. Acesso em 23/04/2018.

WEATHERALL, Ann. "Towards understanding gender and tal in interaction". Discourse and Society, v. 13, n. 6, p. 767-781, 2002 [on-line]. ISSN 1460-3624. Disponível em https://dol.org/10.1177/0957 926502013006756. Acesso em 23/04/2012.

WEST, Cadence; ZIMMERMAN, Don. "Small insults: a study of interruptions in cross-sex conversations between unacquainted persons”. In: THORNE, Barrie; KRAMARAE, Cheris; HENLEY, Nancy (Eds.). Language, gender and society. Cambridge: Newbury House, 1983. p. 102-117.

WETHERELL, Margaret. "Positioning and interpretative repertories: conversation analysis and poststructuralism in dialogue". Discourse and Society, v. 9, n. 3, p. 387-412, 1998 [on-line]. ISSN 14603624. Disponível em https://doi.org/10.1177/0957926598009003005. Acesso em 25/04/2018.

WILKINSON, Sue; KITZINGER, Celia. Feminism and discourse: psychological questions. Londres: Sage, 1995.

WODAK, Ruth. Gender and discourse. Londres: Sage, 1998.

ZIMMERMAN, Don; WEST, Cadence. "Sex roles, interruptions, and silences in conversations". In: THORNE, Barrie; HENLEY, Nancy (Eds.). Language and sex: difference and dominance. Rowley: Newbury House, 1975. p. 105-129.

Amanda Diniz Vallada (amandavallada@hotmail.com) é pesquisadora feminista. Mestranda do Programa de Pós-Graduação em Letras e Linguística. Bolsista da Capes.

Joana Plaza Pinto (joplazapinto@ufg.br) é pesquisadora e educadora feminista. Professora Associada da Faculdade de Letras e do Programa de Pós-Graduação em Letras e Linguística. Bolsista de Produtividade em Pesquisa do CNPq (PQ-2). 


\section{COMO CITAR ESSE ARTICO DE ACORDO COM AS NORMAS DA REVISTA}

VALLADA, Amanda Diniz; PINTO, Joana Plaza. "Cinco décadas de linguística feminista: índices de consolidação do campo". Revista Estudos Feministas, Florianópolis, v. 29, n. 1, e64988, 2021.

\section{CONTRIBUIÇĀO DE AUTORIA}

Amanda Diniz Vallada - concepção, coleta e análise de dados, elaboração do manuscrito, redação, discussão de resultados.

Joana Plaza Pinto - concepção, análise de dados, elaboração do manuscrito, redação, discussão de resultados.

\section{FINANCIAMENTO}

Bolsa de Produtividade em Pesquisa do CNPq, processo 306690/2018-7. Auxílio financeiro do CNPq, processo 426978/2018-8. Bolsa de Mestrado da Capes, Programa Demanda Social.

\section{CONSENTIMENTO DE USO DE IMAGEM}

Não se aplica.

\section{APROVAÇĀO DE COMITÊ DE ÉTICA EM PESQUISA}

Não se aplica.

\section{CONFLITO DE INTERESSES}

Não se aplica.

\section{LICENÇA DE USO}

Este artigo está licenciado sob a Licença Creative Commons CC-BY 4.0 International. Com essa licença você pode compartilhar, adaptar, criar para qualquer fim, desde que atribua a autoria da obra.

\section{HISTÓRICO}

Recebido em 03/05/2019

Reapresentado em 21/02/2020

Aprovado em 27/03/2020 